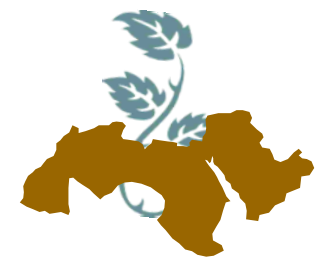

Arab Univ.

J. Agric. Sci., Ain Shams Univ., Cairo, 24(2), 593-608, 2016

\title{
IMPACT OF CALCIUM, HOT WATER AND MODIFIED ATMOSPHERE TREATMENTS IN CHILLING INJURY SUSCEPTIBILITY OF STORED PAPAYAS
}

\author{
Korkar, H.M. \\ Higher Institute for Agric .Co- Operation, Cairo, Egypt
}

Keywords: Solo papayes, Chilling injury, Cold, $\mathrm{CaCl} 2$, Hot water. MAP, Storability

\begin{abstract}
Mature-green solo papaya fruits with color index green with trace of yellow were subjected at 2014 and 2015 seasons to many pre storage treatments. $\mathrm{CaCl} 25 \%$ solution either at ambient temperature or $45^{\circ} \mathrm{C}$ in combined with modified atmosphere package (MAP), hot water at $45^{\circ} \mathrm{C}$ with or without MAP and untreated fruits were investigated, comparing to commercial treatments . All treatments except treatments were cold stored at 5 for one or two weeks followed by 7 days at $18^{\circ} \mathrm{C}$ for marketing period, where commercial treatment was stored at $10^{\circ} \mathrm{C}$ for two weeks and 7 days at $18^{\circ} \mathrm{C}$. The obtained result were recorded at chilling injury symptoms, weight loss \% , decay fruit \% , fruit firmness $(\mathrm{Kg} \backslash \mathrm{f})$., $\mathrm{L}$ - ascorbic acid content T.S.S. ,acidity, salute leakage ethylene production with or without MAP were effective in minimizing orb prevent chilling injury symptoms of solo papaya fruits stored at $5 \pm 1^{\circ} \mathrm{C}$ followed marketing at $18 \pm 1^{\circ} \mathrm{C}$. Storability and good quality and improved chemical characteristics of solo papayas were obtained with pre - storage treatments of solo papaya were obtained with pre - storage treatments compared with control or commercial treatment.
\end{abstract}

\section{INTRODUCTION}

Papaya (Carica papaya L.) is one of the most important fruit crops grown in the tropical and subtropical regions of the world. Being a climacteric fruit, Papaya fruit is highly perishable, and is susceptible to fungal infections, physiological disorders and over-ripeness (Paull el al 1997), papaya has a short postharvest life, thus, research has focused on minimising postharvest losses in order to prolong shelf life.

Papaya is very sensitive to chilling injury $(\mathrm{Cl})$, which limits storage temperature to $8^{\circ} \mathrm{C}$ or above (Chen and Paull 1986 and Ali et al 2004). On the other hand, without refrigeration, papaya discolors and deteriorates in a few days as a result of rapid ripening and disease infection. The postharvest storage procedures for papaya help to minimise the quality deterioration from the decomposition processes (Rohani, et al 1997). The short storage life limits the export of papaya in refrigerated sea containers. Air freight is expensive and shipping in refrigerated containers and modified atmosphere packaging cannot yet offer sufficient storage life without the use of fungicides. Moreover, prolonged storage at low temperatures may damage the fruit physiologically (Maharaj \& Sankat, 1990). If these limitations are minimised, the farmers and exporters of papaya fruit can earn maximum profits and capture a larger share of the world market. Alternative methods that prolong the postharvest storage life of papaya during shipping are required. Recently, there have been increased efforts to discover new preservative compounds derived from natural sources. It is now established that, low temperatures stimulate autocalalytic ethylene production in climacteric fruits (Lelievre et al 1995), and that chilling induced ethylene production follows the general pathway of ethylene biosynthesis (Yang and Hoffman 1994). Various methods such as heat treatment, storage in modified and con- 
trolled atmospheres, intermittent warming, and application of plant growth regulator have been developed to alleviate $\mathrm{Cl}$ symptoms (GonzalezAguilar et al 2001).

Calcium chloride is essential elements, regarding the biological and physiological functions falls largely into five categories, cell wall, membranes, chromosomes, enzyme activation and caphytohormone interaction (Rensburg and Englbrecht, 1986). Also, Ca has received considerable attention, not only because of its relationship to physiological disorders, but also because of other desirable effects, particularly in fruits, where it can reduced the pre climacteric and climacteric respiratory rate, reduce ethylene production, delay ripening, extend storage life, increase firmness and vitamin $\mathrm{C}$ content and reduce storage rot (Eaks, 1985). Calcium content in the harvested fruits plays also vital roles in ethylene biosynthesis and action, respiration and fruit sensitivity to chilling injury which will be discussed later in the postharvest deterioration factors (Yang and Hoffman, 1994) calcium apparently strengthens cell walls and cell membranes and helps tissues withstand chilling stress (Van Eeden, 1992 and Wang, 1994).

The role of calcium in stabilising cellular membranes and delaying senescence in horticultural crops is well known (Poovaiah, et al 1998). Preand post- harvest application of calcium may delay senescence in commercial and retail storage of fruit with no detrimental effect on consumer acceptance (Lester and Grusak, 2004).

Post-harvest heat treatment applied prior to low temperature storage can reduce the incidence of chilling injury in cold-sensitive fruits, such as avocado (Woolf et al 1995) mango (McCollum et al 1993). Heat treatments (HTS), with both hot air and hot water, can also be used for disinfestations (Lurie, 1998). However, HTS can alter several components of fruit quality. Hot water treatment (HWT) can be more easily applied commercially than hot air treatments, particularly if the duration of treatment is short. HWT reduced $\mathrm{Cl}$ after storage but did not eliminate it and the responses were variable (Grove et al 2000). Fruits and vegetables commonly tolerate temperature of $50-60^{\circ} \mathrm{C}$ for $5-10$ minutes (McCollum et al 1993). Heat treatments can affect postharvest physiology, ripening processes and fruit quality (Koukounaras et al 2008) Modified atmosphere is a conservation technique that is used to expand a plant product's postharvest life and to maintain its quality. The main goal of using modified atmosphere is to create an equil- ibrated atmosphere inside packages that is beneficial to the product without being injurious (Kader, 2002). Modified atmosphere have been used on many types of products (Rai et al 2011). Achieving an equilibrium atmosphere inside a package of vegetables and fruits is related to the product's respiration rate, weight, storage temperature, relative humidity, and the permeability characteristics of the packaging material. (Fonseca et al 2002).

The modified atmosphere (MA) method is a good alternative because it requires low investment and technology level, and therefore it has been adopted by small and medium, producers for the preservation of perishable goods (Flores et al 2004). Moreover, this technique allows for longer term storage while maintaining product quality and provides a barrier against insect attacks (Lurie et al 2006). This preservation method leads to a reduction in the fruit respiration rate because the combination of fruit respiration and the gas permeability of the plastic film increases the $\mathrm{CO} 2$ levels and decreases the oxygen $\left(\mathrm{O}_{2}\right)$ inside the package. Thus, there is a change in the metabolic processes (Rocha et al 2004) that slows fruit ripening, microbial growth, moisture loss and enzymatic browning (Guan \& Dou, 2010). Indeed, depending on the levels of fruit respiration and the film permeability, there may be an increase in the CO 2 levels that leads to anaerobic respiration, ethanol accumulation and physiological injuries to the product. This process can result in the production of off-flavors and pulp deterioration, which result in a fruit that is unfit for consumption (Fante et al 2014).

Therefore, the aim of this study was to determine the effects of postharvest application of calcium chloride, hot water and modified atmosphere treatments on the changes in properties related to papaya quality when applied at the light yellow with green coloring stage during prolonged storage.

\section{MATERIALS AND METHODS}

Mature-green solo papaya fruits (Carica papaya L.) with color index green with trace of yellow were harvested in the first week of April during 2014 and 2015. The fruits were harvestd at early morning and directly transferred to post harvest laboratory in Department of Horticulture, Faculty of Agriculture, Ain Shams University, Cairo, Egypt. Papaya fruits used for this experiment were in good appearance and initially had a firmness of 8.4-8.8 Kg/force and soluble solids content ranged from 8.6 to 9.5.Selected fruits were uniform in size, 
shape, and maturity and free from defects. The fruits were sorted, dry cleaned and randomized divided into different treatments. Payaya fruits were then washed with chlorinated water chlorex (0.05\% Sodium hypochlorite) then air dried followed by immersion in Biohealth fungicide with 2.5 $\mathrm{g} / \mathrm{l}$ concentration as fast dipping for all fruits then air dried and wrapped in silky paper. Fruits were weighed for recorded their the initial fresh weight and divided among the following treatments:

1. Fruit dipping in $5 \% \mathrm{CaCl}_{2}$ solution (at ambient temperature) for 5-minutes without packaged polyethylene lining.

2. Fruit dipping in $5 \% \mathrm{CaCl}_{2}$ solution (at ambient temperature) for 5-minutes with packaged polyethylene lining.

3. Fruit dipping in $5 \% \mathrm{CaCl}_{2}$ hot solution (at $45^{\circ} \mathrm{C}$ ) for 5-minutes hot solution of without packaged polyethylene lining.

4. Fruit dipping in $5 \% \mathrm{CaCl}_{2}$ hot solution (at $45^{\circ} \mathrm{C}$ ) for 5-minutes hot solution of with packaged polyethylene lining.

5. Fruit dipped in hot water at $45^{\circ} \mathrm{C}$ for 5 -minutes without packaged polyethylene lining.

6. Fruit dipped in hot water at $45^{\circ} \mathrm{C}$ for 5 -minutes with packaged polyethylene lining.

7. Untreated fruits without packaged polyethylene lining (Control treatment).

8. Untreated fruits with packaged polyethylene lining (Control treatment).

All the above mentioned treatments for papaya fruit were stored for one and two weeks at $5 \pm 1^{\circ} \mathrm{C}$ followed by holding for 7 days at $18 \pm 2^{\circ} \mathrm{C}$ for monitoring and recording the impact of different treatments on the ripening process and quality after transferring fruits from cold store

1. Commercial treatments, fruits were stored at $10 \pm 1^{\circ} \mathrm{C}$ for one or two weeks before transferred to ripening room at $18 \pm 2^{\circ} \mathrm{C}$. (This condition is recommended for commercial storage). Fruits were physically and chemically analyzed after one or two weeks of cold storage at $5 \pm 1^{\circ} \mathrm{C}$ or $10 \pm 1^{\circ} \mathrm{C}$ and 7 days at $18 \pm 2^{\circ} \mathrm{C}$. The fruit of each treatment were arranged in 3 replicates and each was packed in carton boxes with dimensions of $(40 \times 30 \times 10 \mathrm{~cm})$ and each box contained 6 fruit.

\section{Fruit physical analysis}

\subsection{Visual chilling injury symptoms}

Symptoms of chilling injury $(\mathrm{Cl})$ were visually assessed upon removal of the fruits from cold stor- age and threshold. The degree of $\mathrm{Cl}$ severity, based on external damage, such as surface pitting, water-soaked areas and abnormal ripening, was determined in (15 fruits), sample of each treatment, According to (Lederman et al 1997), the modified classifications were as follow:

Scale $1=$ Fruit of free $\mathrm{Cl}$ Symptoms $(0 \%$ surface discoloration)

Scale $2=$ Fruits of very slight $\mathrm{Cl}$ symptoms ( $>0<25 \%$ surface discoloration)

Scale $3=$ Fruits of slight $\mathrm{Cl}$ symptoms (>25<50\% surface discoloration)

Scale $4=$ Fruits of moderate $\mathrm{Cl}$ symptoms ( $>50<75 \%$ surface discoloration)

Scale $5=$ Fruits of severe $\mathrm{Cl}$ symptoms $(>75 \%$ surface discoloration);

The $\mathrm{Cl}$ index was calculated according to the following formula:

\section{Injury level $X$ Number of fruits at the level \\ Cl index = \\ Total number of fruits in the treatment}

\subsection{Percentage of Fruit weight loss (WL\%)}

Fruits initial weight was recorded before chilling treatments and fruit weight loss was calculated by weighing the same fruits at the end of cold storage durations at $5+1^{\circ} \mathrm{C}$ and the end of subsequent storage at $18 \pm 2^{\circ} \mathrm{C}$. (after 7 days) using the following formula:

\section{Fruit initial weight - fruit weight at each sampling date \\ $W L \%=\frac{\text { Fruit initial weight }}{}$ $X 100$}

\subsection{Percentage of decayed fruits}

Fruits showed any sign of decay development by chilling termination and during the subsequent storage period were counted and the percentage of decayed fruits was calculated on the basis of fruits number.

\subsection{Fruit firmness $(\mathrm{Kg} / \mathrm{f})$}

Fruit firmness was determined by peeling the fruit at two equatorial sites and measuring firmness by means of a Wagner® Fruit Firmness Tester, 
model FT-327, equipped with an $8 \mathrm{~mm}$ plunger tip. Values were expressed in kilo gram force $(\mathrm{kg} / \mathrm{f})$. after treatments and immediately after cold storage and the days subsequent storage at $18+2^{\circ} \mathrm{C}$.

\section{Fruit chemical analysis}

\subsection{Respiration rate $(\mathrm{mg} \mathrm{CO} / \mathrm{kg}$ fruits $/ \mathrm{h}$.)}

Carbon dioxide produced by fruits were determined after $10 \mathrm{hrs}$ finished from treatments and after (stored for one or two week and 7 days at 18 $\pm 2^{\circ} \mathrm{C}$ ) The air-flow was passed through concentrated $\mathrm{NaOH}$, to insure that air-flow is $\mathrm{CO}_{2}$ free, before passing into 1-liter jar fruit container (fruit ambient) one fruit/jar was considered as one replicate. The out-coming air-flow was then passed into $100 \mathrm{ml} \mathrm{NaOH}$ of $0.1 \mathrm{~N}$ for $1 \mathrm{~h}$. Such solution was then titrated against 0.1 N HCl (A.O.A.C 2005) and $\mathrm{CO}_{2}$ levels produced by the fruits were then calculated as $\mathrm{mg} \mathrm{CO} 2 / \mathrm{kg}$ fruits $/ \mathrm{h}$.

\subsection{Ethylene production measurements}

Levels of ethylene production (treatments $T_{2} \&$ $T_{4} \& T_{6} \& T_{8}$ and control) analysis by fruits were analyzed after $10 \mathrm{hrs}$ finished from treatments and after (stored for one or two week and 7 days at 18 $\pm 2^{\circ} \mathrm{C}$ ) . Fruits were incubated in glass jars of 1000 $\mathrm{ml}$ volume with a capacity of one fruit per jar. After 2 hrs of incubation, one $\mathrm{ml}$ gas sample was with drawn from each jar head space and injected in a Varian Gas Chromatography model Vestia-6000 with a Flame Ionization Detector (FID) for determining levels of ethylene production. Data were then recorded as $\mathbf{M u ~} \mathbf{C 2} \mathbf{H} \mathbf{4} / \mathbf{K g}$ fresh weight fruit /h of papayas

\subsection{Solute leakage (\% EC leakage)}

Ten grams disks of the fruits tissues were placed in a 100-ml glass beaker containing $30 \mathrm{ml}$ of deionized water and magnetic stirred for $15 \mathrm{~min}$. Electrical conductivity (EC) of the stirrered solution was measured using electrical conductivity meter. Stirrered solution of each beaker was then replaced by equal volume $(30 \mathrm{ml})$ of deionized water for homogenizing the disks in a blender, and the aliquot was then used for measuring EC level as previously described. Percentage of solute leakage was then calculated as EC leakage using the following formula:

\subsection{Total soluble solids (T.S.S \%)}

20 grams of fruit tissues were homogenized in a blender. The homogenized tissues were filtered using What man No. 1 filter paper. The clear juice was decanted and used for T.S.S and titralable acidity analysis. Using hand refractometer, TSS \% was measured using drops of the above extracted juice.

\subsection{Titratable acidity (TA \%)}

By titrating $10 \mathrm{ml}$ of fruit the above extracted juice against $0.1 \mathrm{~N}$ of $\mathrm{NaOH}$ using phenol phathalin indicator according to the Official Methods of Analysis (A.O.A.C 2005). Titratable acidity was expressed as percentage of citric acid ( $\mathrm{g}$ citric ac$\mathrm{id} / 100 \mathrm{ml}$ juice).

\subsection{L-Ascorbic acid ( $\mathrm{mg} / 100 \mathrm{~g}$ fresh weight)}

The L-ascorbic acid content (V.C) was determined and expressed as $\mathrm{mg} / 100 \mathrm{~g}$ fresh weight using $10 \mathrm{~g}$. of the fruit tissues were homogenized in $100 \mathrm{ml}$ of $3 \%$ oxalic acid, the extraction was filtered and $10 \mathrm{ml}$ was titrated against 2,6-dichlo phenolendo phenol dye following the methods by (A.O.A.C. 2005).

\section{Statistical analysis}

Data obtained in the two studied seasons were conducted in a Completely Randomized Design (CRD) with three replications. Data obtained were subjected to Analysis of variance (ANOVA) using MSTAT-C software (MSTAT Michigan University East Lansing). Duncan multiple rang test (LSR) was performed to determine any significant difference among various treatments. $p<0.05$ was selected as decision for significant differences) according to (Steel et al 1997).

\section{RESULTS AND DISCUSSION}

\section{Physical determinations}

\subsection{Chilling injury (scale)}

Data tabulated in Table (1) showed that, appearing of chilling injury symptoms greatly affected with different treatments. However dipping solo 

in chilling injury susceptibility of stored Papayas

papaya fruits in $\mathrm{CaCl} 25 \%$ solution either at ambient temperature or at $45^{\circ} \mathbf{C}$ with or without modified atmosphere packing (MAP) was effective in store the fruits at $5{ }^{\circ} \mathrm{C}$ without $\mathrm{Cl}$ symptoms (1. 0 scale). This finding was similar commercial storage at $10^{\circ} \mathrm{C}$ for one or two weeks which means that it could be stored solo papaya fruits at $5^{\circ} \mathrm{C}$ after pre storage treated with $\mathrm{CaCl} 2$ application. On the other hand, untreated solo papaya fruits either with (MAP) or without exhibited 1.9 \& 1.9 scale after one week and $2.4 \& 2.5$ scale after two week at $5^{\circ} \mathrm{C}$. This finding of results was nearly found for the both studied seasons. However, chilling injury symptoms was development at $18+2{ }^{\circ} \mathrm{C}$ (marketable period for 7 days but the positive effect to mentioned treatment still appear

It is well known that low temperature stresses render the commodity more susceptible to postharvest pathogens and sensitivity chilling injury (Paull, 1990). It has been observed in many chilling-sensitive crops that modification of the atmosphere surrounding the commodity and the high humidity created in-package, suppresses $\mathrm{Cl}$ (Gonza'lez-Aguilar et al 2003) Calcium apparently strengthens cell walls and cell membranes and helps tissue withstand chilling stress. It has reduced chilling injury (Wang 1994 \& McDonald, 1996).

\subsection{Weight loss $\%$}

Generally, an observed increase in weight loss $\%$ was recorded with increasing time of cold storage and the following days to $18 \pm 2^{\circ} \mathrm{C}$ (Table 2). It is clear that packaging fruits in polyethylene line led to a reduction weight loss\% regardless of the combined treatments.

Weight loss of polyethylene packaged fruits after two weeks of cold storage ranged (from 2.06 to $3.88 \%$ ) at first season and (from 2.50 to $4.01 \%$ ) at second season compared to untreated fruits and non packaged which recorded (5.80 and $4.97 \%$ ) during both seasons after chilled fruits for two weeks. Packaged hot calcium chloride treated fruits led also to a reduction in weight loss than those treated with same treatment with non- packaged polyethylene line. On the contrary untreated fruits and hot water treated without packaged polyethylene line fruits were exhibited highest values of weight loss \% than other treatments or control. Gonza'lez-Aguilar et al (2003) reported that weight losses greater than $8 \%$ considerably diminished the postharvest quality of papaya.
Post-harvest dipping in $\mathrm{Ca}^{++}$was effective in terms of membrane functionality and integrity maintenance, with lower losses of phospholipids and proteins and reduced ion leakage and respiration rate (Eaks, 1985) therefore reduced weight loss. (Nasr et al 2004) Shrink wrapping have been reported to reduce weight loss of papaya fruit (Lazan et al 1990).

\subsection{Fruits decay $\%$}

An evident increase in fruits decay \% after the 7 days in temperate at $18 \pm 2^{\circ} \mathrm{C}$, especially with the fruit chilled for two weeks irrespective of the used treatments (Table 3).

Slight differences between treatments of $\mathrm{CaCl}_{2}$ were noticed, however, hot $\mathrm{CaCl}_{2}$ plus polyethylene packaged $\left(T_{4}\right)$ were exhibited the least values of decay fruit comparing with commercial treatment (T9) fruits which give values (6.67 and $13.34 \%$ ) at the end of period (7days) subsequent of cold storage for two weeks during 2014 and 2015 seasons, respectively. No significant difference between treated fruits with calcium chloride with packaged and the hot calcium chloride without packaged which gave values of fruit decay ranging from 20.0 to $26.67 \%$ during both season after two weeks of cold storage. On the other side, untreated fruit (control) and hot water treated fruits without packaged recorded the highest percentage of decay fruits with no significant differences between them. After 7 days at $18 \pm 2^{\circ} \mathrm{C}$ under two season decay percentage reached to (33.34 \& $33.34 \%)$ with chilled fruit for one weeks and (46.67 \& $53.00 \%)$ with chilled fruits for two weeks during both seasons.

Calcium chloride treatment resulted in a reduction of rot decay. Beneficial effects of calcium against postharvest decay have been shown for various fruit species (Sams, et al 1993). Gallerani et al (1990) reported that calcium not only protects apple from the physiological disorders, but also help in reducing decay, by strengthening the cell wall and regulating the metabolic activity in the fruit.

\subsection{Fruit firmness $(\mathrm{Kg} /$ force)}

A clear decreased in fruit firmness of solo papaya fruits during storage durations (either at cold storage or subsequent days at $18 \pm 2^{\circ} \mathrm{C}$ with all used treatment (Table 4). $\mathrm{CaCl}_{2}$ application slowly fruit firmness loss (reduced fruit softness) during the subsequent storage at $18 \pm 2^{\circ} \mathrm{C}$ specially 
Table 1. Effect of calcium, hot water and modified atmosphere treatments on chilling injures of solo papaya fruits during storage at $5 \pm 1{ }^{\circ} \mathrm{C}$ (treat. 1-8) and at $10 \pm 11^{\circ} \mathrm{C}$ (treat.9) for one or two week followed by 7 days at $18 \pm 2^{\circ} \mathrm{C}$ during 2014 and 2015 seasons

\begin{tabular}{|c|c|c|c|c|c|c|c|c|}
\hline \multirow{3}{*}{ 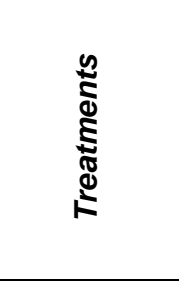 } & \multicolumn{4}{|c|}{2014 season } & \multicolumn{4}{|c|}{2015 season } \\
\hline & \multicolumn{2}{|c|}{$\begin{array}{l}\text { Cold storage for } \\
\text { one week }\end{array}$} & \multicolumn{2}{|c|}{$\begin{array}{l}\text { Cold storage for } \\
\text { two week }\end{array}$} & \multicolumn{2}{|c|}{$\begin{array}{l}\text { Cold storage for } \\
\text { one week }\end{array}$} & \multicolumn{2}{|c|}{$\begin{array}{l}\text { Cold storage for } \\
\text { two week }\end{array}$} \\
\hline & $\begin{array}{l}\text { Out of } \\
\text { storage }\end{array}$ & $\begin{array}{c}7 \text { days } \\
\text { at } 18 \pm \\
2^{\circ} \mathrm{C}\end{array}$ & $\begin{array}{l}\text { Out of } \\
\text { storage }\end{array}$ & $\begin{array}{c}7 \text { days } \\
\text { at } 18 \pm \\
2^{\circ} \mathrm{C} \\
\end{array}$ & $\begin{array}{l}\text { Out of } \\
\text { storage }\end{array}$ & $\begin{array}{c}7 \text { days } \\
\text { at } 18 \pm \\
2^{\circ} \mathrm{C} \\
\end{array}$ & $\begin{array}{l}\text { Out of } \\
\text { storage }\end{array}$ & $\begin{array}{c}7 \text { days } \\
\text { at } 18 \pm \\
2^{\circ} \mathrm{C} \\
\end{array}$ \\
\hline $\mathrm{CaCl}_{2}{ }^{\mathrm{a}}$ & $1.0 \mathrm{~b}$ & $1.4 \mathrm{~b}$ & $1.3 \mathrm{c}$ & $1.8 \mathrm{~cd}$ & $1.0 \mathrm{~b}$ & $1.4 \mathrm{c}$ & $1.3 \mathrm{bc}$ & $1.7 \mathrm{~cd}$ \\
\hline $\mathrm{CaCl}_{2}{ }^{\mathrm{b}}$ & $1.0 \mathrm{~b}$ & $1.3 \mathrm{~b}$ & $1.0 \mathrm{c}$ & $1.4 \mathrm{~d}$ & $1.0 \mathrm{~b}$ & $1.1 \mathrm{c}$ & $1.0 \mathrm{c}$ & $1.4 \mathrm{~d}$ \\
\hline Hot $\mathrm{CaCl}_{2}{ }^{\mathrm{a}}$ & $1.0 \mathrm{~b}$ & $1.1 \mathrm{~b}$ & $1.0 \mathrm{c}$ & $1.3 \mathrm{~d}$ & $1.0 \mathrm{~b}$ & $1.2 \mathrm{c}$ & $1.0 \mathrm{c}$ & $1.4 \mathrm{~d}$ \\
\hline Hot $\mathrm{CaCl}_{2}{ }^{\mathrm{b}}$ & $1.0 \mathrm{~b}$ & $1.0 \mathrm{~b}$ & $1.0 \mathrm{c}$ & $1.2 \mathrm{~d}$ & $1.0 \mathrm{~b}$ & $1.0 \mathrm{c}$ & $1.0 \mathrm{c}$ & $1.1 \mathrm{~d}$ \\
\hline Hot water ${ }^{a}$ & $1.6 \mathrm{~b}$ & $2.2 \mathrm{a}$ & $2.3 a b$ & $2.9 \mathrm{~b}$ & $1.4 a b$ & $2.1 \mathrm{ab}$ & $1.8 \mathrm{ab}$ & $2.6 \mathrm{~b}$ \\
\hline Hot water ${ }^{b}$ & $1.4 a b$ & $1.9 \mathrm{a}$ & $1.8 \mathrm{~b}$ & $2.3 \mathrm{bc}$ & $1.3 \mathrm{ab}$ & $1.7 \mathrm{~b}$ & $1.5 a b$ & $2.2 \mathrm{bc}$ \\
\hline Untreated $^{a}$ & $1.9 \mathrm{a}$ & $2.3 \mathrm{a}$ & $2.4 \mathrm{a}$ & $3.6 \mathrm{a}$ & $1.7 \mathrm{a}$ & $2.3 \mathrm{a}$ & $2.0 \mathrm{a}$ & $3.2 \mathrm{a}$ \\
\hline Untreated $^{\mathrm{b}}$ & $1.9 \mathrm{a}$ & $2.4 \mathrm{a}$ & $2.5 \mathrm{a}$ & $3.5 a b$ & $1.5 \mathrm{ab}$ & $2.5 \mathrm{a}$ & $1.9 \mathrm{a}$ & $3.4 \mathrm{a}$ \\
\hline Control & $1.0 \mathrm{~b}$ & $1.0 \mathrm{~b}$ & $1.0 \mathrm{c}$ & $1.0 \mathrm{c}$ & $1.0 \mathrm{~b}$ & $1.0 \mathrm{c}$ & $1.0 \mathrm{c}$ & $1.0 \mathrm{~d}$ \\
\hline
\end{tabular}

Values followed by the same letter in each column are not statistically different at $5 \%$ level

${ }^{a}$ Fruits without packaged polyethylene ${ }^{\mathrm{b}}$ Fruits packaged polyethylene lining

${ }^{*}$ Fruit Chilling injures grade are $: 1$. free \& 2. very slight \& 3. slight \& 4. moderate and 5. sever

Table 2. Effect of calcium , hot water and modified atmosphere treatments on fruit weight loss $\%$ of solo papaya fruits during storage at $5 \pm 1{ }^{\circ} \mathrm{C}$ (treat. 1-8) and at $10 \pm 1{ }^{\circ} \mathrm{C}$ (treat.9) for one or two week followed by 7 days at $18 \pm 2^{\circ} \mathrm{C}$ during 2014 and 2015 seasons

\begin{tabular}{|c|c|c|c|c|c|c|c|c|}
\hline \multirow{3}{*}{ Treat. } & \multicolumn{4}{|c|}{2014 season } & \multicolumn{4}{|c|}{2015 season } \\
\hline & \multicolumn{2}{|c|}{$\begin{array}{l}\text { Cold storage for } \\
\text { one week }\end{array}$} & \multicolumn{2}{|c|}{$\begin{array}{l}\text { Cold storage for } \\
\text { two week }\end{array}$} & \multicolumn{2}{|c|}{$\begin{array}{l}\text { Cold storage for } \\
\text { one week }\end{array}$} & \multicolumn{2}{|c|}{$\begin{array}{l}\text { Cold storage for } \\
\text { two week }\end{array}$} \\
\hline & $\begin{array}{l}\text { Out of } \\
\text { storage }\end{array}$ & $\begin{array}{c}7 \text { days } \\
\text { at } 18 \pm \\
2^{\circ} \mathrm{C}\end{array}$ & $\begin{array}{l}\text { Out of } \\
\text { storage }\end{array}$ & $\begin{array}{c}7 \text { days } \\
\text { at } 18 \pm \\
2^{\circ} \mathrm{C}\end{array}$ & $\begin{array}{l}\text { Out of } \\
\text { storage }\end{array}$ & $\begin{array}{c}7 \text { days } \\
\text { at } 18 \pm \\
2^{\circ} \mathrm{C}\end{array}$ & $\begin{array}{l}\text { Out of } \\
\text { storage }\end{array}$ & $\begin{array}{c}7 \text { days } \\
\text { at } 18 \pm \\
2^{\circ} \mathrm{C}\end{array}$ \\
\hline $\mathrm{CaCl}_{2}{ }^{\mathrm{a}}$ & $1.22 \mathrm{bc}$ & $2.34 \mathrm{c}$ & $1.44 \mathrm{~cd}$ & $3.31 \mathrm{~cd}$ & 1.33ab & $2.61 d$ & $1.80 \mathrm{c}$ & $3.28 \mathrm{c}$ \\
\hline $\mathrm{CaCl}_{2}{ }^{\mathrm{b}}$ & $0.91 \mathrm{~cd}$ & $2.06 \mathrm{~cd}$ & $1.14 \mathrm{~d}$ & $2.84 \mathrm{de}$ & $0.94 \mathrm{~cd}$ & $2.16 \mathrm{e}$ & $1.34 \mathrm{~d}$ & $2.71 \mathrm{~d}$ \\
\hline Hot $\mathrm{CaCl}_{2}{ }^{\mathrm{a}}$ & $1.16 \mathrm{bc}$ & $1.97 \mathrm{~cd}$ & $1.36 \mathrm{~cd}$ & $2.67 \mathrm{de}$ & $1.25 b$ & $2.34 \mathrm{de}$ & $1.95 \mathrm{bc}$ & $3.02 \mathrm{~cd}$ \\
\hline Hot $\mathrm{CaCl}_{2}{ }^{\mathrm{b}}$ & $0.81 \mathrm{~d}$ & $1.71 \mathrm{~d}$ & $1.11 \mathrm{~d}$ & $2.06 \mathrm{e}$ & $0.76 d$ & $1.82 \mathrm{e}$ & $1.18 \mathrm{~d}$ & $2.50 \mathrm{~d}$ \\
\hline Hot water ${ }^{a}$ & $1.65 \mathrm{a}$ & $4.15 \mathrm{a}$ & $2.11 a b$ & $4.85 a b$ & $1.49 \mathrm{ab}$ & $3.65 \mathrm{~b}$ & $2.47 a b$ & $4.80 \mathrm{a}$ \\
\hline Hot water ${ }^{b}$ & $1.03 \mathrm{~cd}$ & $3.34 \mathrm{~b}$ & $1.53 \mathrm{~cd}$ & $3.88 \mathrm{bc}$ & $1.21 b c$ & $3.21 \mathrm{~d}$ & $1.73 \mathrm{c}$ & $4.01 \mathrm{~b}$ \\
\hline Untreated $^{a}$ & $1.55 \mathrm{a}$ & $4.56 \mathrm{a}$ & $2.54 \mathrm{a}$ & $5.66 \mathrm{a}$ & $1.62 \mathrm{a}$ & $4.05 \mathrm{a}$ & $2.62 \mathrm{a}$ & $5.11 \mathrm{a}$ \\
\hline Untreated $^{\text {b }}$ & $1.41 \mathrm{ab}$ & $4.42 a$ & $1.85 \mathrm{bc}$ & $5.80 \mathrm{a}$ & $1.53 \mathrm{a}$ & $4.39 \mathrm{a}$ & $1.97 \mathrm{bc}$ & $4.97 \mathrm{a}$ \\
\hline Control & $1.35 \mathrm{ab}$ & $3.54 \mathrm{~b}$ & $1.47 \mathrm{~cd}$ & $3.88 \mathrm{bc}$ & $1.17 b c$ & $2.94 \mathrm{~cd}$ & $1.56 \mathrm{~cd}$ & $3.94 \mathrm{~b}$ \\
\hline
\end{tabular}

Values followed by the same letter in each column are not statistically different at $5 \%$ level

${ }^{a}$ Fruits without packaged polyethylene ${ }^{b}$ Fruits packaged polyethylene lining 

in chilling injury susceptibility of stored Papayas

Table 3. Effect of calcium, hot water and modified atmosphere treatments on decay percentage of solo papaya fruits during storage at $5 \pm 1^{\circ} \mathrm{C}$ (treat. 1-8) and at $10 \pm 1^{\circ} \mathrm{C}$ (treat.9) for one or two week followed by 7 days at $18 \pm 2^{\circ} \mathrm{C}$ during 2014 and 2015 seasons

\begin{tabular}{|c|c|c|c|c|c|c|c|c|}
\hline \multirow{3}{*}{ 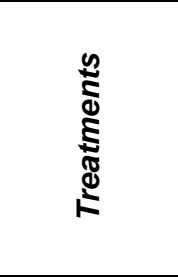 } & \multicolumn{4}{|c|}{2014 season } & \multicolumn{4}{|c|}{2015 season } \\
\hline & \multicolumn{2}{|c|}{$\begin{array}{l}\text { Cold storage for } \\
\text { one week }\end{array}$} & \multicolumn{2}{|c|}{$\begin{array}{l}\text { Cold storage for } \\
\text { two week }\end{array}$} & \multicolumn{2}{|c|}{$\begin{array}{l}\text { Cold storage for } \\
\text { one week }\end{array}$} & \multicolumn{2}{|c|}{$\begin{array}{l}\text { Cold storage for } \\
\text { two week }\end{array}$} \\
\hline & $\begin{array}{l}\text { Out of } \\
\text { storage }\end{array}$ & $\begin{array}{c}7 \text { days } \\
\text { at } 18 \pm \\
2^{\circ} \mathrm{C}\end{array}$ & $\begin{array}{l}\text { Out of } \\
\text { storage }\end{array}$ & $\begin{array}{c}7 \text { days } \\
\text { at } 18 \pm \\
2^{\circ} \mathrm{C}\end{array}$ & $\begin{array}{l}\text { Out of } \\
\text { storage }\end{array}$ & $\begin{array}{c}7 \text { days } \\
\text { at } 18 \pm \\
2^{\circ} \mathrm{C}\end{array}$ & $\begin{array}{l}\text { Out of } \\
\text { storage }\end{array}$ & $\begin{array}{c}7 \text { days } \\
\text { at } 18 \pm \\
2^{\circ} \mathrm{C}\end{array}$ \\
\hline $\mathrm{CaCl}_{2}{ }^{\mathrm{a}}$ & $0.0 \mathrm{~b}$ & $13.3 \mathrm{~cd}$ & $6.67 \mathrm{bc}$ & $26.67 \mathrm{bc}$ & $0.0 \mathrm{a}$ & $20.0 \mathrm{bc}$ & $0.0 \mathrm{c}$ & $26.67 \mathrm{c}$ \\
\hline $\mathrm{CaCl}_{2}{ }^{\mathrm{b}}$ & $0.0 \mathrm{~b}$ & $6.7 \mathrm{~d}$ & $0.0 \mathrm{c}$ & $20.0 \mathrm{bc}$ & $0.0 \mathrm{a}$ & $13.34 \mathrm{~cd}$ & $0.0 \mathrm{c}$ & $26.67 \mathrm{c}$ \\
\hline Hot $\mathrm{CaCl}_{2}{ }^{\mathrm{a}}$ & $0.0 \mathrm{~b}$ & $6.7 \mathrm{~d}$ & $0.0 \mathrm{c}$ & $20.0 \mathrm{bc}$ & $0.0 \mathrm{a}$ & $6.7 \mathrm{~d}$ & $0.0 \mathrm{c}$ & $20.0 \mathrm{c}$ \\
\hline Hot $\mathrm{CaCl}_{2}{ }^{\mathrm{b}}$ & $0.0 \mathrm{~b}$ & $0.0 \mathrm{~d}$ & $0.0 \mathrm{c}$ & $6.67 \mathrm{~d}$ & $0.0 \mathrm{a}$ & $6.7 \mathrm{~d}$ & $0.0 \mathrm{c}$ & $13.34 \mathrm{~cd}$ \\
\hline Hot water ${ }^{a}$ & $6.67 \mathrm{ab}$ & $26.7 a b$ & $13.34 \mathrm{ab}$ & $46.7 \mathrm{a}$ & $0.0 \mathrm{a}$ & $33.34 \mathrm{a}$ & $6.7 \mathrm{bc}$ & $53.00 \mathrm{a}$ \\
\hline Hot water ${ }^{b}$ & $0.0 \mathrm{~b}$ & $20.0 \mathrm{bc}$ & $6.67 \mathrm{bc}$ & $33.34 a b$ & $0.0 \mathrm{a}$ & $26.67 \mathrm{ab}$ & $13.34 a b$ & $40.00 \mathrm{~b}$ \\
\hline Untreated $^{a}$ & $6.67 \mathrm{ab}$ & $26.7 \mathrm{ab}$ & $20.0 \mathrm{a}$ & $46.7 \mathrm{a}$ & $6.67 \mathrm{a}$ & $33.34 \mathrm{a}$ & $20.0 a$ & $53.00 \mathrm{a}$ \\
\hline Untreated $^{\text {b }}$ & $13.34 \mathrm{a}$ & $33.3 \mathrm{a}$ & $20.0 \mathrm{a}$ & $46.7 \mathrm{a}$ & $6.67 \mathrm{a}$ & $33.34 \mathrm{a}$ & $20.0 \mathrm{a}$ & $46.67 \mathrm{ab}$ \\
\hline control & $0.0 \mathrm{~b}$ & $0.0 \mathrm{~d}$ & $0.0 \mathrm{c}$ & $13.34 \mathrm{~cd}$ & $0.0 \mathrm{a}$ & $0.0 \mathrm{~d}$ & $0.0 \mathrm{c}$ & $6.67 \mathrm{~d}$ \\
\hline
\end{tabular}

Values followed by the same letter in each column are not statistically different at $5 \%$ level

${ }^{a}$ Fruits without packaged polyethylene ${ }^{b}$ Fruits packaged polyethylene lining

Table 4. Effect of calcium, hot water and modified atmosphere treatments Fruit firmness (Kg/ force) of solo papaya fruits during storage at $5 \pm 1^{\circ} \mathrm{C}$ (treat. 1-8) and at $10 \pm 1^{\circ} \mathrm{C}$ (treat.9) for one or two week followed by 7 days at $18 \pm 2^{\circ} \mathrm{C}$ during 2014 and 2015 seasons

\begin{tabular}{|c|c|c|c|c|c|c|c|c|}
\hline \multirow{3}{*}{ 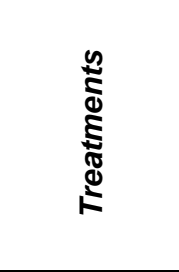 } & \multicolumn{4}{|c|}{2014 season } & \multicolumn{4}{|c|}{2015 season } \\
\hline & \multicolumn{2}{|c|}{$\begin{array}{l}\text { Cold storage for } \\
\text { one week }\end{array}$} & \multicolumn{2}{|c|}{$\begin{array}{l}\text { Cold storage for } \\
\text { two week }\end{array}$} & \multicolumn{2}{|c|}{$\begin{array}{l}\text { Cold storage for } \\
\text { one week }\end{array}$} & \multicolumn{2}{|c|}{$\begin{array}{l}\text { Cold storage for } \\
\text { two week }\end{array}$} \\
\hline & $\begin{array}{l}\text { Out of } \\
\text { storage }\end{array}$ & $\begin{array}{c}7 \text { days } \\
\text { at } 18 \pm \\
2^{\circ} \mathrm{C}\end{array}$ & $\begin{array}{l}\text { Out of } \\
\text { storage }\end{array}$ & $\begin{array}{c}7 \text { days } \\
\text { at } 18 \pm \\
2^{\circ} \mathrm{C}\end{array}$ & $\begin{array}{l}\text { Out of } \\
\text { storage }\end{array}$ & $\begin{array}{c}7 \text { days } \\
\text { at } 18 \pm \\
2^{\circ} \mathrm{C}\end{array}$ & $\begin{array}{c}\text { Out of } \\
\text { storage }\end{array}$ & $\begin{array}{c}7 \text { days } \\
\text { at } 18 \pm \\
2^{\circ} \mathrm{C} \\
\end{array}$ \\
\hline $\mathrm{CaCl}_{2}{ }^{\mathrm{a}}$ & $6.0 \mathrm{bc}$ & $5.5 a b$ & $5.6 \mathrm{bc}$ & $5.1 b$ & $6.1 \mathrm{bc}$ & $5.7 b$ & $5.8 b c$ & $5.1 b$ \\
\hline $\mathrm{CaCl}_{2}{ }^{\mathrm{b}}$ & $6.0 b c$ & $5.4 a b$ & $5.9 \mathrm{ab}$ & $5.2 \mathrm{ab}$ & $6.4 b$ & $6.0 \mathrm{~b}$ & $6.3 a b$ & $5.7 a$ \\
\hline Hot $\mathrm{CaCl}_{2}{ }^{\mathrm{a}}$ & $6.2 b$ & $5.7 a$ & $5.8 \mathrm{bc}$ & $5.3 a b$ & $6.5 b$ & $5.8 b$ & $6.2 \mathrm{ab}$ & $5.7 a$ \\
\hline Hot $\mathrm{CaCl}_{2}{ }^{\mathrm{b}}$ & $7.3 a$ & $5.6 \mathrm{ab}$ & $6.4 a$ & $5.8 a$ & $7.8 \mathrm{a}$ & $7.0 \mathrm{a}$ & $6.8 \mathrm{a}$ & $6.1 \mathrm{a}$ \\
\hline Hot water ${ }^{a}$ & $5.6 \mathrm{~cd}$ & $5.0 \mathrm{c}$ & $5.4 \mathrm{~cd}$ & $4.1 \mathrm{~cd}$ & $6.0 \mathrm{bc}$ & $4.6 d$ & $5.0 \mathrm{~cd}$ & $4.0 \mathrm{~cd}$ \\
\hline Hot water ${ }^{b}$ & $5.8 b c$ & $5.3 b$ & $5.5 \mathrm{bc}$ & $4.3 c$ & $5.7 \mathrm{c}$ & $4.9 \mathrm{~cd}$ & $5.0 \mathrm{~cd}$ & $4.5 \mathrm{c}$ \\
\hline Untreated $^{\mathrm{a}}$ & $5.3 d$ & 4.6d & $5.1 d$ & $3.7 d$ & $5.5 \mathrm{c}$ & $4.3 d$ & $4.8 d$ & $4.2 \mathrm{~cd}$ \\
\hline Untreated $^{\text {b }}$ & $5.3 d$ & $4.7 \mathrm{~cd}$ & $5.2 d$ & $3.9 \mathrm{~cd}$ & $5.6 c$ & 4.4d & $4.6 d$ & $3.7 d$ \\
\hline control & $5.9 \mathrm{bc}$ & $5.4 a b$ & $5.6 \mathrm{bc}$ & $5.1 \mathrm{~b}$ & $6.0 \mathrm{bc}$ & $5.2 \mathrm{c}$ & $5.4 \mathrm{c}$ & $5.0 \mathrm{~b}$ \\
\hline
\end{tabular}

Values followed by the same letter in each column are not statistically different at $5 \%$ level

${ }^{a}$ Fruits without packaged polyethylene ${ }^{\mathrm{b}}$ Fruits packaged polyethylene lining

Initial sample recorded 8.6 and $9.1 \mathrm{~kg} /$ force at first and second season 
the fruits packed with polyethylene. Slight differences between $\mathrm{CaCl}_{2}$ treatment plus packed $\left(\mathrm{T}_{2}\right)$ and hot calcium chloride treatment without packed $\left(T_{3}\right)$ which give $(5.2 \& 5.3 \& 5.7$ and $5.7 \mathrm{Kg} /$ force) during both seasons after 7 days at $18 \pm 2^{\circ} \mathrm{C}$ subsequent two weeks of cold storage respectively. The less values of fruit firmness were obtained with untreated and hot water treated fruits for 1 and 2 weeks. A sharp decrease in fruit firmness were noticed with untreated and hot water treated fruits only without significant differences between them which reached (3.7\& 3.9 and $4.1 \mathrm{Kg} /$ force) during 2014 and $(3.7 \& 4.2$ and $4.0 \mathrm{Kg} /$ force) during 2015 after two weeks chilled fruit, storage.

Heat-treatment of fruit most often increases firmness and reduces softening during postharvest storage (Valero et al 2002). Inhibition of solubilisation of the carbonate-soluble pectin fraction is one of the main factors contributing to the firmness retention due to heat treatment (Lurie, et al 2006).

The effect of calcium in tissue firmness is generally explained by complexing cell wall and middle lamella polygalacturonic acid residues imparting improvement of structural integrity (Morris, 1980). However, calcium ions may also impact tissue firmness by contributing to increase membrane integrity and the consequent maintenance or increase of cell turgor pressure (Mahmud, et al 2008).

\section{Chemical determinations}

\subsection{Respiration Rate (mg CO2/kg-hr)}

It is clear, (Table, 5) that respiration rate was decreased at the end of cold storage durations (one or two weeks) with all used treatments compared with commercial storage of fruits (fruits stored at $10 \pm 1^{\circ} \mathrm{C}$ ) which recorded (13.71 and 12.0 $\mathrm{mg} \mathrm{CO}_{2} / \mathrm{kg}$-hr) at first and second respectively.

$\mathrm{CaCl}_{2}$ treatments led to a reduction of respiration rate after one and two weeks at cold storage and after 7 days holding at the $\left(18 \pm 2^{\circ} \mathrm{C}\right)$ compared to control treatment.

However, the packed fruits in polyethylene line led to a reduction in respiratory rate coefficients within same treatments $\left(T_{2}\right.$ and $\left.T_{4}\right)$ which recorded (26.3 and $25.1 \mathrm{mg} \mathrm{CO} / \mathrm{kg}$ fruit $/ \mathrm{hr}$. ) at first season and (21.5 and $25.4 \mathrm{mg} \mathrm{CO} / \mathrm{kg}$ fruit $/ \mathrm{hr}$.) at second season after 7 days at $18 \pm 2^{\circ} \mathrm{C}$ for two weeks chilling fruit respectively. Untreated or hot water treated fruits exhibited a decrease in respiration rate during cold storage followed by a sharp in- crease after removal from cold storage and during holding at $18 \pm 2^{\circ} \mathrm{C}$ ranged from (35.4 to 38.2 and34.9 to $39.1 \mathrm{mg} \mathrm{CO} / \mathrm{kg}$ fruit $/ \mathrm{hr}$ ) at first and second seasons receptively after two weeks at $(5 \pm$ $1^{\circ} \mathrm{C}$ ) and subsequent 7 days at $18 \pm 2^{\circ} \mathrm{C}$.

$\mathrm{Ca}^{++}$dipping reduced fruit sensitivity to chilling injury sine it reduced the respiration and plasma membrane degradation (Sabehat, et al 1998). Calcium inhibited both the preclimacteric and climacteric respiratory rates (Chávez-Sánchez et al 2013. The effective treatments in minimizing respiration rate was $\mathrm{CaCl}_{2}$ plus hot water. (Nasr, 2004), The rate of oxidation relative to glycolysis which may lead to the accumulation of an intermediate respiratory substances to toxic levels or fermentation causing chilling injury development (Sabehat, et al 1998).

\subsection{Ethylene production ( $\mathrm{ppm} / \mathrm{g}$ fruit fresh weight/hr}

Data tabulated in Table (6) showed the effect of different treatments on ethylene production of solo papaya fruits after one and two weeks of cold storage at $5 \pm 1^{\circ} \mathrm{C}$ and subsequent 7 days at $18 \pm$ $2^{\circ} \mathrm{C}$. Generally, ethylene production was decreased during cold storage durations with all used treatments compared with initial sample. At the end of cold storage duration (two weeks) calcium chloride treatments either alone or in combination with hot water caused a gradual increase in ethylene production during subsequent days of cold storage. An evident increase in ethylene production during days of storage at $18 \pm 2{ }^{\circ} \mathrm{C}$ with all used treatments. Hot calcium chloride with fruit packed of polyethylene line achieved the highest values of ethylene production which recorded (4.35 and 4.43 $\mathrm{mu} / \mathrm{kg}$ fruit fresh weight/hr) during 2014 and 2015 seasons in the end of subsequent days after two weeks of chilled fruit at $5 \pm 1^{\circ} \mathrm{C}$ respectively .

On contrary, untreated recorded the least values of ethylene production.A sharp decrease in ethylene production during cold storage caused a general reduction in ethylene production which means that fruits failure in ripening, which recorded $(1.71$ and $1.62 \mathrm{mu} / \mathrm{kg}$ fruit fresh weight/hr) during 2014 and 2015 seasons in the end of subsequent days after two weeks of chilled fruit at 5 $\pm 1^{\circ} \mathrm{C}$ respectively .

Ethylene production and the capacity to convert ACC to ethylene were not enhanced by storage temperatures, which cause chilling injury to the peel (Lederman et al 1997). Lower biosynthesis 
ethylene action inside the package during storage is dependent on the action of this plant growth regulator (Argenta et al 2003 and Fante et al 2014).

\subsection{Total soluble solids (TSS \%)}

Generally, it could be noticed that total soluble solids (Table 7) were increased with advanced in cold storage duration regardless of pre-storage treatments. Initial samples recorded (9.1 and 8.9 $\%$ ), while commercial fruits recorded (12.2 \& 12.6 \&12.3 and $12.5 \%$ ) in the end of days at $18 \pm 2^{\circ} \mathrm{C}$ at both season under study of chilled fruits for one and two weeks at $5 \pm 11^{\circ} \mathrm{C}$ respectively. No significant differences between all used treatments on T.S.S\% were noticed after the end of both durations of cold storage ( $1 \& 2$ weeks). The differences between treatments were appeared during its presence in the temperature at $18 \pm 2^{\circ} \mathrm{C}$. The highest T.S.S\% were obtained with $\mathrm{CaCl}_{2}$-treated fruits with hot water in combined with packed in polyethyen line during both seasons. An observed reduction of total soluble solids with untreated and hot water treated fruits without packed were more sensitive to chilling injury which recorded values ranged from 10.7 to 11 and from 10.5 to $10.8 \%$ in the end of days at $18 \pm 2^{\circ} \mathrm{C}$ after chilled fruits of two weeks at $5 \pm 1^{\circ} \mathrm{C}$ during first and second seasons

\subsection{Titratable acidity $\%$}

As shown in Table (8), hot calcium chloride treatment with packaged polyethylene lining exhibited decreasing in acidity which give values $(0.18$ and $0.20 \%$ ) during both seasons after 7 days subsequence the two weeks respectively. No significant differences between $\mathrm{CaCl}_{2}$ with packaged polyethylene lining and hot $\mathrm{CaCl}_{2}$ only treatments which gave moderate values of acidity. On the other hand, untreated chilled fruits for two weeks showing symptoms of $\mathrm{Cl}$ as well as a significant increase in ttitratable acidity (TA) compared to other treatments and control which reached to $0.32 \%$ at first season and $0.37 \%$ at second season. The inhibition of such enzymes as a resulted of $\mathrm{Cl}$ may resulted in accumulation of their substances which are acidic metabolic substances. The accumulations of such middle substances result in the subsequent development of $\mathrm{Cl}$ symptoms. Since most of this middle substances are acids, it is logic therefore, to assume that higher acidity level will be paralleled to the fruits having symptoms of $\mathrm{Cl}$. It is suggested, therefore, that acidity increment is an accurate indicator for fruit $\mathrm{Cl}$. This phenomenon is unique, practical and need to be tested on other fruit species sensitive to $\mathrm{Cl}$. (Nasr, 2004).

Retarding the papaya fruit ripening by calcium causing inhibition of enzyme activity could explain the delay in the use of organic acids in the enzymatic reactions of respiration. Organic acids in papaya are largely citric and malic acids and the increase in $\mathrm{pH}$ during ripening and storage was due to the metabolic processes of the fruit that resulted in adecrease of the organic acids. (Mahmud et al 2008).

\subsection{L-ascorbic acid content $(\mathrm{mg} / 100 \mathrm{~g}$ fresh weight)}

It is clear (Table 9), decrease that a L-ascorbic acid content of solo papaya fruits during storage durations (cold storage and subsequent days at $18 \pm 2^{\circ} \mathrm{C}$ in both seasons. An evident decline in rate of L-ascorbic acid content in chilled fruits for two weeks than chilled fruits for one week. No significant differences in the end of cold storage among all tested treatments after one week however, differences began to appear in the end of two weeks of chilled fruits. Hot calcium chloride treatments (with or without MAP) exhibited the high values of $\mathrm{L}$-ascorbic acid compared to other treatments. Untreated contained less values of L-ascorbic than others. The reduction in L-ascorbic acid in the untreated fruits were ranged from $(32.4 \mathrm{mg}$ to 16.3 $\mathrm{mg} / 100 \mathrm{~g}$ fresh weight (about $49.69 \%$ losses in Lascorbic acid) at first season and (34.3 $\mathrm{mg}$ to 18.7 $\mathrm{mg} / 100 \mathrm{~g}$ fresh weight (about $45.35 \%$ losses in Lascorbic acid) at second season (from initial sample to the end of subsequent days of two weeks at cold storage).

Ascorbic acid contents for papaya, during cold storage start to decline Lazan et al (1990) and Mathooko (1996) reported that the ascorbic acid content in tomato increased with maturity and ripening. However, once fruit became fully ripe, the vitamin content started to decline. $\mathrm{CaCl}_{2}$ treated fruit were firmer and had more ascorbic acid than untreated fruit (Sams et al 1993).

\subsection{Solute leakage \% (EC)}

An evident increase in solute leakage Table (10) with increasing time of cold storage and the following days to $18 \pm 2^{\circ} \mathrm{C}$ with all treated or untreated fruits. It is noted that also packaging fruits with polyethylene (MAP) has reduced solute 
Table 5. Effect of calcium , hot water and modified atmosphere treatments on respiration rate (mg $\mathrm{CO} 2 / \mathrm{Kg}$ fruit $/ \mathrm{hr}$.) of solo papaya fruits during storage at $5 \pm 1^{\circ} \mathrm{C}$ (treat. 1-8) and at $10 \pm 1^{\circ} \mathrm{C}$ (treat.9) for one or two week followed by 7 days at $18 \pm 2^{\circ} \mathrm{C}$ during 2014 and 2015 seasons

\begin{tabular}{|c|c|c|c|c|c|c|c|c|}
\hline \multirow{3}{*}{ 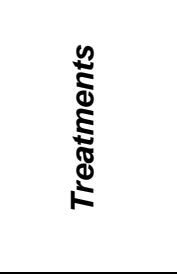 } & \multicolumn{4}{|c|}{2014 season } & \multicolumn{4}{|c|}{2015 season } \\
\hline & \multicolumn{2}{|c|}{$\begin{array}{l}\text { Cold storage for } \\
\text { one week }\end{array}$} & \multicolumn{2}{|c|}{$\begin{array}{l}\text { Cold storage for } \\
\text { two week }\end{array}$} & \multicolumn{2}{|c|}{$\begin{array}{l}\text { Cold storage for } \\
\text { one week }\end{array}$} & \multicolumn{2}{|c|}{$\begin{array}{l}\text { Cold storage for } \\
\text { two week }\end{array}$} \\
\hline & $\begin{array}{l}\text { Out of } \\
\text { storage }\end{array}$ & $\begin{array}{c}7 \text { days } \\
\text { at } 18 \pm \\
2^{\circ} \mathrm{C}\end{array}$ & $\begin{array}{l}\text { Out of } \\
\text { storage }\end{array}$ & $\begin{array}{c}7 \text { days } \\
\text { at } 18 \pm \\
2^{\circ} \mathrm{C}\end{array}$ & $\begin{array}{l}\text { Out of } \\
\text { storage }\end{array}$ & $\begin{array}{c}7 \text { days } \\
\text { at } 18 \pm \\
2^{\circ} \mathrm{C}\end{array}$ & $\begin{array}{l}\text { Out of } \\
\text { storage }\end{array}$ & $\begin{array}{c}7 \text { days } \\
\text { at } 18 \pm \\
2^{\circ} \mathrm{C}\end{array}$ \\
\hline $\mathrm{CaCl}_{2}{ }^{\mathrm{a}}$ & $6.96 \mathrm{de}$ & $24.2 \mathrm{~b}$ & $7.6 \mathrm{~cd}$ & $29.7 c$ & $6.5 \mathrm{~cd}$ & $19.3 b c$ & $7.3 \mathrm{~cd}$ & $27.9 \mathrm{c}$ \\
\hline $\mathrm{CaCl}_{2}{ }^{\mathrm{b}}$ & $5.64 e$ & $19.6 \mathrm{c}$ & $6.4 \mathrm{~d}$ & $26.3 \mathrm{~d}$ & $5.2 \mathrm{~cd}$ & $16.1 \mathrm{de}$ & $6.3 \mathrm{~d}$ & $25.4 d$ \\
\hline Hot $\mathrm{CaCl}_{2}{ }^{\mathrm{a}}$ & 6.83de & $23.7 \mathrm{~b}$ & $7.7 \mathrm{~cd}$ & $30.1 \mathrm{c}$ & $6.0 \mathrm{~cd}$ & $17.0 \mathrm{~cd}$ & $6.9 \mathrm{~cd}$ & 28.1c \\
\hline Hot $\mathrm{CaCl}_{2}{ }^{\mathrm{b}}$ & $5.91 \mathrm{e}$ & $18.7 \mathrm{c}$ & $6.2 \mathrm{~d}$ & $25.1 \mathrm{~d}$ & $4.9 \mathrm{~d}$ & $14.1 \mathrm{e}$ & $5.6 \mathrm{~d}$ & $21.5 \mathrm{e}$ \\
\hline Hot water ${ }^{a}$ & $8.10 \mathrm{~cd}$ & $29.5 \mathrm{a}$ & 9.4 bc & $37.6 \mathrm{a}$ & $7.5 b$ & $26.1 \mathrm{a}$ & $8.9 b c$ & $36.3 \mathrm{~b}$ \\
\hline Hot water ${ }^{b}$ & $7.30 c-e$ & $23.4 \mathrm{~b}$ & $8.3 \mathrm{~cd}$ & $35.4 \mathrm{~b}$ & $6.4 \mathrm{~cd}$ & $20.7 \mathrm{~b}$ & $7.4 \mathrm{~cd}$ & $34.9 \mathrm{~b}$ \\
\hline Untreated $^{\mathrm{a}}$ & $10.87 b$ & 29.4 a & $11.7 \mathrm{~b}$ & $38.2 \mathrm{a}$ & $9.7 \mathrm{~b}$ & $27.9 a$ & $10.2 b$ & 37.3ab \\
\hline Untreated $^{\mathrm{b}}$ & $9.24 b c$ & $29.9 \mathrm{a}$ & $10.6 \mathrm{bc}$ & $37.4 \mathrm{ab}$ & $9.0 \mathrm{~b}$ & $28.6 \mathrm{a}$ & $9.5 \mathrm{~b}$ & $39.1 \mathrm{a}$ \\
\hline Control & $13.71 \mathrm{a}$ & 24.7 b & $18.7 \mathrm{a}$ & $30.6 \mathrm{c}$ & $12.0 \mathrm{a}$ & $21.9 b$ & $16.9 \mathrm{a}$ & $27.1 \mathrm{~cd}$ \\
\hline
\end{tabular}

Values followed by the same letter in each column are not statistically different at $5 \%$ level

${ }^{a}$ Fruits without packaged polyethylene ${ }^{\mathrm{b}}$ Fruits packaged polyethylene lining

Initial sample recorded: and $\mathrm{kg} /$ force at first and second season

Table 6. Effect of calcium, hot water and modified atmosphere treatments on Ethylene production $\mathrm{Mu} \mathrm{C2H4} / \mathrm{Kg}$ fresh weight fruit $/ \mathrm{h}$ of solo papaya fruits during storage at $5 \pm 1^{\circ} \mathrm{C}$ (treat. 1-8) and at $10 \pm$ $1^{\circ} \mathrm{C}$ (treat.9) for one or two week followed by 7 days at $18 \pm 2^{\circ} \mathrm{C}$ during 2014 and 2015 seasons

\begin{tabular}{|c|c|c|c|c|c|c|c|c|}
\hline \multirow{3}{*}{ 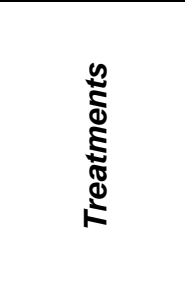 } & \multicolumn{4}{|c|}{2014 season } & \multicolumn{4}{|c|}{2015 season } \\
\hline & \multicolumn{2}{|c|}{$\begin{array}{l}\text { Cold storage for } \\
\text { one week }\end{array}$} & \multicolumn{2}{|c|}{$\begin{array}{l}\text { Cold storage for } \\
\text { two week }\end{array}$} & \multicolumn{2}{|c|}{$\begin{array}{l}\text { Cold storage for } \\
\text { one week }\end{array}$} & \multicolumn{2}{|c|}{$\begin{array}{l}\text { Cold storage for } \\
\text { two week }\end{array}$} \\
\hline & $\begin{array}{l}\text { Out of } \\
\text { storage }\end{array}$ & $\begin{array}{c}7 \text { days } \\
\text { at } 18 \pm \\
2^{\circ} \mathrm{C} \\
\end{array}$ & $\begin{array}{l}\text { Out of } \\
\text { storage }\end{array}$ & $\begin{array}{c}7 \text { days } \\
\text { at } 18 \pm \\
2^{\circ} \mathrm{C}\end{array}$ & $\begin{array}{l}\text { Out of } \\
\text { storage }\end{array}$ & $\begin{array}{c}7 \text { days } \\
\text { at } 18 \pm \\
2^{\circ} \mathrm{C} \\
\end{array}$ & $\begin{array}{l}\text { Out of } \\
\text { storage }\end{array}$ & $\begin{array}{c}7 \text { days } \\
\text { at } 18 \pm \\
2^{\circ} \mathrm{C} \\
\end{array}$ \\
\hline $\mathrm{CaCl}_{2}$ & $1.21 \mathrm{~b}$ & $3.74 a$ & $0.98 b$ & 3.11b & $1.11 b$ & $3.35 \mathrm{~b}$ & $1.07 \mathrm{~b}$ & $3.59 \mathrm{~b}$ \\
\hline $\mathrm{CaCl}_{2}+\mathrm{Hot}$ & $1.35 \mathrm{ab}$ & $3.92 a$ & $1.05 b$ & $4.35 a$ & $1.45 a$ & $3.84 a$ & $1.17 \mathrm{~b}$ & $4.43 a$ \\
\hline Hot water & $0.77 \mathrm{c}$ & $2.45 b$ & $0.61 \mathrm{c}$ & $2.08 \mathrm{c}$ & $0.87 b c$ & $2.51 \mathrm{c}$ & $0.63 c$ & $1.72 \mathrm{c}$ \\
\hline Untreated & $0.65 c$ & $1.98 \mathrm{c}$ & $0.57 \mathrm{c}$ & $1.71 \mathrm{~d}$ & $0.59 c$ & $1.88 \mathrm{~d}$ & $0.53 \mathrm{c}$ & $1.62 \mathrm{c}$ \\
\hline Control & $1.58 \mathrm{a}$ & $3.69 \mathrm{a}$ & $2.13 a$ & $4.56 \mathrm{a}$ & $1.74 a$ & $3.93 a$ & $2.41 \mathrm{a}$ & $4.40 a$ \\
\hline
\end{tabular}

Values followed by the same letter in each column are not statistically different at $5 \%$ level

${ }^{a}$ Fruits without packaged polyethylene ${ }^{\mathrm{b}}$ Fruits packaged polyethylene lining

Initial sample recorded 1.21 and $1.546 \mathrm{mu} / \mathrm{kg} \mathrm{Kg}$ fresh weight fruit/ hr force at first and second season 

in chilling injury susceptibility of stored Papayas

Table 7. Effect of calcium and modified atmosphere treatments on total soluble solids (S.S.C. \%) of solo papaya fruits during storage at $5 \pm 1^{\circ} \mathrm{C}$ (treat. 1-8) and at $10 \pm 1^{\circ} \mathrm{C}$ (treat.9) for one or two week followed by 7 days at $18 \pm 2^{\circ} \mathrm{C}$ during 2014 and 2015 seasons

\begin{tabular}{|c|c|c|c|c|c|c|c|c|}
\hline \multirow{3}{*}{ 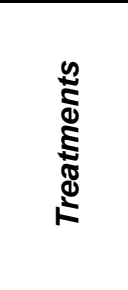 } & \multicolumn{4}{|c|}{2014 season } & \multicolumn{4}{|c|}{2015 season } \\
\hline & \multicolumn{2}{|c|}{$\begin{array}{l}\text { Cold storage for } \\
\text { one week }\end{array}$} & \multicolumn{2}{|c|}{$\begin{array}{l}\text { Cold storage for } \\
\text { two week }\end{array}$} & \multicolumn{2}{|c|}{$\begin{array}{l}\text { Cold storage for } \\
\text { one week }\end{array}$} & \multicolumn{2}{|c|}{$\begin{array}{l}\text { Cold storage for } \\
\text { two week }\end{array}$} \\
\hline & $\begin{array}{l}\text { Out of } \\
\text { storage }\end{array}$ & $\begin{array}{c}7 \text { days } \\
\text { at } 18 \pm \\
2^{\circ} \mathrm{C}\end{array}$ & $\begin{array}{l}\text { Out of } \\
\text { storage }\end{array}$ & $\begin{array}{c}7 \text { days } \\
\text { at } 18 \pm \\
2^{\circ} \mathrm{C}\end{array}$ & $\begin{array}{l}\text { Out of } \\
\text { storage }\end{array}$ & $\begin{array}{c}7 \text { days } \\
\text { at } 18 \pm \\
2^{\circ} \mathrm{C}\end{array}$ & $\begin{array}{l}\text { Out of } \\
\text { storage }\end{array}$ & $\begin{array}{c}7 \text { days } \\
\text { at } 18 \pm \\
2^{\circ} \mathrm{C}\end{array}$ \\
\hline$T_{1}{ }^{a}$ & $10.0 \mathrm{a}$ & $12.0 \mathrm{a}$ & $10.6 \mathrm{a}$ & $12.4 \mathrm{a}$ & $9.4 \mathrm{a}$ & $12.1 \mathrm{a}$ & $10.7 \mathrm{a}$ & $12.0 \mathrm{ab}$ \\
\hline $\mathbf{T}_{2}{ }^{b}$ & $10.1 \mathrm{a}$ & $12.0 \mathrm{a}$ & $10.7 \mathrm{a}$ & $12.2 \mathrm{ab}$ & $9.3 \mathrm{a}$ & $12.2 \mathrm{a}$ & $10.6 \mathrm{a}$ & $12.2 \mathrm{a}$ \\
\hline $\mathbf{T}_{3}{ }^{\mathrm{a}}$ & $10.3 \mathrm{a}$ & $12.0 \mathrm{a}$ & $10.5 \mathrm{a}$ & 12.4 a & $9.6 \mathrm{a}$ & $12.0 \mathrm{a}$ & $10.8 \mathrm{a}$ & $12.3 \mathrm{a}$ \\
\hline$T_{4}{ }^{b}$ & $10.2 \mathrm{a}$ & $12.1 \mathrm{a}$ & $10.6 \mathrm{a}$ & $12.5 \mathrm{a}$ & $9.5 \mathrm{a}$ & $12.1 \mathrm{a}$ & $10.9 \mathrm{a}$ & $12.2 \mathrm{a}$ \\
\hline $\mathbf{T}_{5}{ }^{\mathrm{a}}$ & $10.1 \mathrm{a}$ & $12.2 \mathrm{a}$ & $10.6 \mathrm{a}$ & $10.7 \mathrm{c}$ & $9.3 \mathrm{a}$ & $11.6 \mathrm{a}$ & $10.6 \mathrm{a}$ & $10.8 \mathrm{c}$ \\
\hline$T_{6}{ }^{b}$ & $10.4 \mathrm{a}$ & $12.1 \mathrm{a}$ & $10.7 \mathrm{a}$ & $11.3 \mathrm{bc}$ & $9.4 \mathrm{a}$ & $11.6 \mathrm{a}$ & $10.7 \mathrm{a}$ & $11.0 \mathrm{bc}$ \\
\hline$T_{7}^{a}$ & $10.3 \mathrm{a}$ & $11.8 \mathrm{a}$ & $10.5 \mathrm{a}$ & $11.0 \mathrm{c}$ & $9.3 \mathrm{a}$ & $11.3 \mathrm{a}$ & $10.5 \mathrm{a}$ & $10.5 \mathrm{c}$ \\
\hline$T_{8}^{b}$ & $10.3 \mathrm{a}$ & $11.9 \mathrm{a}$ & $10.7 \mathrm{a}$ & $10.9 \mathrm{c}$ & $9.5 \mathrm{a}$ & $11.4 \mathrm{a}$ & $10.3 \mathrm{a}$ & $10.7 \mathrm{c}$ \\
\hline Control & $10.8 \mathrm{a}$ & $12.2 \mathrm{a}$ & $11.1 \mathrm{a}$ & $12.6 \mathrm{a}$ & 9.9 a & $12.3 \mathrm{a}$ & $10.9 \mathrm{a}$ & $12.5 \mathrm{a}$ \\
\hline
\end{tabular}

Values followed by the same letter in each column are not statistically different at $5 \%$ level

${ }^{a}$ Fruits without packaged polyethylene ${ }^{\mathrm{b}}$ Fruits packaged polyethylene lining

Initial sample recorded 9.5 and $8.9 \mathrm{~kg} /$ force at first and second season

Table 8. Effect of calcium, hot water modified atmosphere treatments on titratable acidity (TA\%) of solo papaya fruits during storage at $5 \pm 1^{\circ} \mathrm{C}$ (treat. 1-8) and at $10 \pm 1^{\circ} \mathrm{C}$ (treat.9) for one or two week followed by 7 days at $18 \pm 2^{\circ} \mathrm{C}$ during 2014 and 2015 seasons

\begin{tabular}{|c|c|c|c|c|c|c|c|c|}
\hline \multirow{3}{*}{ 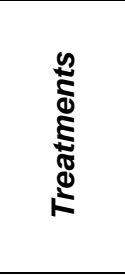 } & \multicolumn{4}{|c|}{2014 season } & \multicolumn{4}{|c|}{2015 season } \\
\hline & \multicolumn{2}{|c|}{$\begin{array}{l}\text { Cold storage for } \\
\text { one week }\end{array}$} & \multicolumn{2}{|c|}{$\begin{array}{l}\text { Cold storage for } \\
\text { two week }\end{array}$} & \multicolumn{2}{|c|}{$\begin{array}{l}\text { Cold storage for } \\
\text { one week }\end{array}$} & \multicolumn{2}{|c|}{$\begin{array}{l}\text { Cold storage for } \\
\text { two week }\end{array}$} \\
\hline & $\begin{array}{l}\text { Out of } \\
\text { storage }\end{array}$ & $\begin{array}{c}7 \text { days } \\
\text { at } 18 \pm \\
2^{\circ} \mathrm{C}\end{array}$ & $\begin{array}{l}\text { Out of } \\
\text { storage }\end{array}$ & $\begin{array}{c}7 \text { days } \\
\text { at } 18 \pm \\
2^{\circ} \mathrm{C} \\
\end{array}$ & $\begin{array}{c}\text { Out of } \\
\text { storage }\end{array}$ & $\begin{array}{c}7 \text { days } \\
\text { at } 18 \pm \\
2^{\circ} \mathrm{C} \\
\end{array}$ & $\begin{array}{c}\text { Out of } \\
\text { storage }\end{array}$ & $\begin{array}{c}7 \text { days } \\
\text { at } 18 \pm \\
2^{\circ} \mathrm{C}\end{array}$ \\
\hline $\mathrm{T}_{1}^{\mathrm{a}}$ & $0.26 \mathrm{a}$ & $0.23 a$ & $0.24 a$ & $0.20 \mathrm{c}$ & $0.31 \mathrm{a}$ & $0.25 \mathrm{a}$ & $0.26 a$ & $0.24 \mathrm{c}$ \\
\hline $\mathbf{T}_{2}{ }^{b}$ & $0.25 a$ & $0.22 \mathrm{a}$ & $0.23 a$ & $0.20 \mathrm{c}$ & $0.29 a$ & $0.26 \mathrm{a}$ & $0.27 \mathrm{a}$ & $0.24 \mathrm{c}$ \\
\hline $\mathbf{T}_{3}{ }^{\mathrm{a}}$ & $0.24 a$ & $0.24 a$ & $0.23 a$ & $0.21 \mathrm{c}$ & $0.28 a$ & $0.25 a$ & $0.26 \mathrm{a}$ & $0.24 \mathrm{c}$ \\
\hline $\mathbf{T}_{4}{ }^{b}$ & $0.26 \mathrm{a}$ & $0.25 \mathrm{a}$ & $0.22 \mathrm{a}$ & $0.18 \mathrm{c}$ & $0.28 \mathrm{a}$ & $0.24 \mathrm{a}$ & $0.25 \mathrm{a}$ & $0.20 \mathrm{c}$ \\
\hline $\mathbf{T}_{5}{ }^{a}$ & $0.25 a$ & $0.24 a$ & $0.26 \mathrm{a}$ & $0.32 \mathrm{a}$ & $0.30 \mathrm{a}$ & $0.26 \mathrm{a}$ & $0.27 a$ & $0.30 \mathrm{~b}$ \\
\hline$T_{6}^{b}$ & $0.26 a$ & $0.23 a$ & $0.25 a$ & $0.28 a b$ & $0.29 a$ & $0.25 a$ & $0.27 \mathrm{a}$ & $0.32 \mathrm{~b}$ \\
\hline $\mathbf{T}_{7}{ }^{\mathrm{a}}$ & $0.25 a$ & $0.25 a$ & $0.24 a$ & $0.31 \mathrm{a}$ & $0.29 a$ & $0.25 a$ & $0.28 a$ & $0.37 a$ \\
\hline $\mathrm{T}_{8}{ }^{\mathrm{b}}$ & $0.26 \mathrm{a}$ & $0.23 \mathrm{a}$ & $0.25 \mathrm{a}$ & $0.31 \mathrm{a}$ & $0.31 \mathrm{a}$ & $0.24 \mathrm{a}$ & $0.29 \mathrm{a}$ & $0.34 a b$ \\
\hline Control & $0.24 \mathrm{a}$ & $0.21 \mathrm{a}$ & $0.20 \mathrm{a}$ & $0.18 \mathrm{c}$ & $0.28 \mathrm{a}$ & $0.23 \mathrm{a}$ & $0.26 \mathrm{a}$ & $0.22 \mathrm{c}$ \\
\hline
\end{tabular}

Values followed by the same letter in each column are not statistically different at $5 \%$ level

${ }^{a}$ Fruits without packaged polyethylene ${ }^{b}$ Fruits packaged polyethylene lining

Initial sample recorded 0.28 and $0.34 \%$ at first and second season 
Table 9. Effect of calcium, hot water and modified atmosphere treatments on L.ascorbic acid content (LAA $\mathbf{~ m g} / \mathbf{1 0 0} \mathbf{g}$ fresh weight) of solo papaya fruits during storage at $5 \pm 1^{\circ} \mathrm{C}$ (treat. $1-8$ ) and at $10 \pm$ $1^{\circ} \mathrm{C}$ (treat.9) for one or two week followed by 7 days at $18 \pm 2^{\circ} \mathrm{C}$ during 2014 and 2015 seasons

\begin{tabular}{|c|c|c|c|c|c|c|c|c|}
\hline \multirow{3}{*}{ 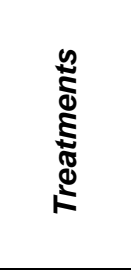 } & \multicolumn{4}{|c|}{2014 season } & \multicolumn{4}{|c|}{2015 season } \\
\hline & \multicolumn{2}{|c|}{$\begin{array}{l}\text { Cold storage for } \\
\text { one week }\end{array}$} & \multicolumn{2}{|c|}{$\begin{array}{l}\text { Cold storage for } \\
\text { two week }\end{array}$} & \multicolumn{2}{|c|}{$\begin{array}{l}\text { Cold storage for } \\
\text { one week }\end{array}$} & \multicolumn{2}{|c|}{$\begin{array}{l}\text { Cold storage for } \\
\text { two week }\end{array}$} \\
\hline & $\begin{array}{l}\text { Out of } \\
\text { storage }\end{array}$ & $\begin{array}{c}7 \text { days } \\
\text { at } 18 \pm \\
2^{\circ} \mathrm{C} \\
\end{array}$ & $\begin{array}{l}\text { Out of } \\
\text { storage }\end{array}$ & $\begin{array}{c}7 \text { days } \\
\text { at } 18 \pm \\
2^{\circ} \mathrm{C}\end{array}$ & $\begin{array}{l}\text { Out of } \\
\text { storage }\end{array}$ & $\begin{array}{c}7 \text { days } \\
\text { at } 18 \pm \\
2^{\circ} \mathrm{C} \\
\end{array}$ & $\begin{array}{l}\text { Out of } \\
\text { storage }\end{array}$ & $\begin{array}{c}7 \text { days } \\
\text { at } 18 \pm \\
2^{\circ} \mathrm{C} \\
\end{array}$ \\
\hline $\mathrm{T}_{1}{ }^{\mathrm{a}}$ & $20.1 \mathrm{c}$ & $25.8 \mathrm{ab}$ & $28.6 a$ & 23.3ab & $31.2 a$ & $28.0 \mathrm{bc}$ & $28.0 \mathrm{a}$ & $22.7 \mathrm{bc}$ \\
\hline $\mathbf{T}_{2}{ }^{b}$ & $29.4 a b$ & 26.3ab & $28.0 \mathrm{ab}$ & $23.5 a b$ & $30.0 \mathrm{ab}$ & $28.4 \mathrm{~b}$ & $27.9 \mathrm{a}$ & $23.8 \mathrm{ab}$ \\
\hline$T_{3}{ }^{a}$ & $29.5 a b$ & $27.1 a$ & $27.8 \mathrm{ab}$ & $24.1 a$ & $29.7 a-c$ & $30.7 a$ & $27.1 \mathrm{ab}$ & 23.9ab \\
\hline$T_{4}^{b}$ & $29.9 a$ & $27.7 a$ & $28.9 a$ & $24.5 a$ & $31.4 a$ & $29.3 a b$ & 29.0 a & $25.1 a$ \\
\hline$T_{5}{ }^{a}$ & $26.9 a b$ & $22.7 \mathrm{~cd}$ & $24.1 \mathrm{~cd}$ & $18.9 c$ & $30.7 a b$ & $25.3 d$ & $24.3 \mathrm{~cd}$ & $19.3 d$ \\
\hline$T_{6}^{b}$ & 27.8ab & $23.9 c$ & $25.3 c$ & $19.2 c$ & $30.5 a b$ & $26.4 \mathrm{~cd}$ & $24.4 \mathrm{~cd}$ & $20.7 \mathrm{~cd}$ \\
\hline $\mathbf{T}_{7}{ }^{a}$ & $26.0 \mathrm{~b}$ & $21.5 d$ & $23.4 \mathrm{~cd}$ & $16.3 d$ & $28.1 \mathrm{c}$ & $25.1 \mathrm{de}$ & $23.1 \mathrm{~d}$ & $19.1 d$ \\
\hline$T_{8}^{b}$ & $26.0 \mathrm{~b}$ & $22.6 \mathrm{~cd}$ & $24.0 \mathrm{~cd}$ & $17.2 \mathrm{~cd}$ & $28.7 \mathrm{bc}$ & $24.0 \mathrm{e}$ & $23.6 \mathrm{~cd}$ & $18.7 d$ \\
\hline Control & 27.3ab & $24.3 b c$ & $26.0 \mathrm{bc}$ & $21.9 b$ & $28.9 b c$ & $27.1 \mathrm{~cd}$ & $25.3 \mathrm{bc}$ & $22.4 \mathrm{bc}$ \\
\hline
\end{tabular}

Values followed by the same letter in each column are not statistically different at $5 \%$ level

${ }^{a}$ Fruits without packaged polyethylene ${ }^{\mathrm{b}}$ Fruits packaged polyethylene lining

Initial sample recorded 32.4 and 34.3 LAA mg/100 g fresh weight at first and second season

Table 10. Effect of calcium , hot water and modified atmosphere treatments on solute leakage \% (EC) of solo papaya fruits during storage at $5 \pm 1^{\circ} \mathrm{C}$ (treat. $1-8$ ) and at $10 \pm 1^{\circ} \mathrm{C}$ (treat.9) for one or two week followed by 7 days at $18 \pm 2^{\circ} \mathrm{C}$ during 2014 and 2015 seasons

\begin{tabular}{|c|c|c|c|c|c|c|c|c|}
\hline \multirow{3}{*}{ 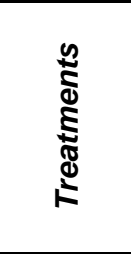 } & \multicolumn{4}{|c|}{2014 season } & \multicolumn{4}{|c|}{2015 season } \\
\hline & \multicolumn{2}{|c|}{$\begin{array}{l}\text { Cold storage for } \\
\text { one week }\end{array}$} & \multicolumn{2}{|c|}{$\begin{array}{l}\text { Cold storage for } \\
\text { two week }\end{array}$} & \multicolumn{2}{|c|}{$\begin{array}{c}\text { Cold storage for } \\
\text { one week }\end{array}$} & \multicolumn{2}{|c|}{$\begin{array}{l}\text { Cold storage for } \\
\text { two week }\end{array}$} \\
\hline & $\begin{array}{l}\text { Out of } \\
\text { storage }\end{array}$ & $\begin{array}{c}7 \text { days } \\
\text { at } 18 \pm \\
2^{\circ} \mathrm{C} \\
\end{array}$ & $\begin{array}{c}\text { Out of } \\
\text { storage }\end{array}$ & $\begin{array}{c}7 \text { days } \\
\text { at } 18 \pm \\
2^{\circ} \mathrm{C}\end{array}$ & $\begin{array}{c}\text { Out of } \\
\text { storage }\end{array}$ & $\begin{array}{c}7 \text { days } \\
\text { at } 18 \pm \\
2^{\circ} \mathrm{C}\end{array}$ & $\begin{array}{c}\text { Out of } \\
\text { storage }\end{array}$ & $\begin{array}{c}7 \text { days } \\
\text { at } 18 \pm \\
2^{\circ} \mathrm{C} \\
\end{array}$ \\
\hline $\mathrm{T}_{1}^{\mathrm{a}}$ & $19.1 \mathrm{bc}$ & $25.1 \mathrm{~cd}$ & 21.4de & $27.9 c$ & $17.5 \mathrm{c}$ & $22.5 \mathrm{~cd}$ & $19.6 c$ & 26.3d \\
\hline $\mathbf{T}_{2}{ }^{b}$ & $19.0 \mathrm{bc}$ & $24.5 d$ & $20.0 \mathrm{e}$ & $25.8 d$ & $17.0 \mathrm{c}$ & $21.4 d$ & $19.1 \mathrm{c}$ & $25.0 d$ \\
\hline$T_{3}{ }^{a}$ & $18.7 c$ & $23.9 d$ & $19.9 e$ & $26.1 \mathrm{~cd}$ & $16.7 \mathrm{c}$ & $21.0 \mathrm{~d}$ & $18.8 \mathrm{c}$ & $24.8 \mathrm{de}$ \\
\hline $\mathbf{T}_{4}{ }^{\mathrm{b}}$ & $17.5 \mathrm{c}$ & $21.8 \mathrm{e}$ & $19.6 e$ & $24.5 d$ & $16.3 c$ & $20.7 d$ & $18.5 c$ & $23.2 e$ \\
\hline$T_{5}{ }^{a}$ & $21.5 a$ & $28.6 b$ & $24.6 b c$ & $35.0 a$ & 19.3ab & $25.8 b$ & $21.9 b$ & $35.2 b$ \\
\hline $\mathbf{T}_{6}{ }^{\mathrm{b}}$ & $21.0 \mathrm{ab}$ & $26.9 b c$ & $23.1 \mathrm{~cd}$ & $32.1 \mathrm{~b}$ & $18.2 b c$ & $24.8 b$ & $20.5 b c$ & $31.5 c$ \\
\hline $\mathrm{T}_{7}^{\mathrm{a}}$ & $22.8 a$ & $30.7 a$ & $34.9 a$ & $35.2 a$ & $20.5 a$ & $29.5 a$ & $25.8 \mathrm{a}$ & $39.9 a$ \\
\hline$T_{8}{ }^{b}$ & $21.5 a$ & $31.4 a$ & $26.0 \mathrm{~b}$ & $36.8 \mathrm{a}$ & $20.9 a$ & $30.3 a$ & $26.7 a$ & $36.4 b$ \\
\hline Control & $22.4 a$ & $24.9 \mathrm{~cd}$ & $23.2 \mathrm{c}$ & $26.6 \mathrm{~cd}$ & $19.5 \mathrm{ab}$ & $23.9 \mathrm{bc}$ & $22.3 b$ & 26.7d \\
\hline
\end{tabular}

Values followed by the same letter in each column are not statistically different at $5 \%$ level

${ }^{a}$ Fruits without packaged polyethylene ${ }^{b}$ Fruits packaged polyethylene lining

Initial sample recorded : and $\mathrm{kg} /$ force at first and second season 

in chilling injury susceptibility of stored Papayas

leakage \% compared to those without packaging. A negative correlation was also found between $\mathrm{CaCl}_{2}$ application with packaged polyethylene line $\left(T_{3}\right)$ and fruit solute leakage parameter during cold storage at $5 \pm 1^{\circ} \mathrm{C}$ and followed by holding for 7 days at $18 \pm 2^{\circ} \mathrm{C}$. which give lower values of solute leakage at 2014 season and at 2015 season of chilled fruits for two weeks. On contrary, untreated fruits or treated with hot water exhibited the highest values of solute leakage result from collapse of a large internal ranged from $31.2 \%$ to $36.8 \%$ at first season and 31.5 to $39.9 \%$ at second season, after two weeks of chilled fruit in the end days $18 \pm 2^{\circ} \mathrm{C}$ respectively. With chilling injury, membrane permeability considerably increased due to its deterioration allowing more solute leakage.

The chilling of sensitive fruit has increased Electrolyte leakage (Lester et al 1988). It is clear that calcium chloride has a role in the protection cell membrane and the protection and suggests that the reason for reducing the fruit solute leakage. Calcium apparently strengthens cell walls and cell membranes and helps tissues withstand chilling stress (Wang, 1994 and Hernandez et al 2006).

\section{REFERENCES}

A.O.A.C. 2005. Official Methods of the Analysis of AOAC. International18th Edition, Published by AOAC International. Maryland 2087-2417. USA.

Ali, Z.M., Chin, L., Marimuthu, M. and Lazan, H. 2004. Low temperature storage and modified atmosphere packaging of carambola fruit and their effects on ripening related texture changes, wall modification and chilling injury symptoms. Postharvest Biology and Technology, 33(2), 181-192.

Argenta, L.C., Krammes, J.G., Megguer, C.A., Amarante, B.C.V. and Mattheis, J. 2003. Ripening and quality of 'Laetitia' plums following harvest and cold storage as affected by inhibition of ethylene action. Pesquisa Agropecuária Brasileira, 38(10), 1139-1148.

Chávez-Sánchez, I., Carrillo-López, A., VegaGarcía, M. and Yahia, E.M. 2013. The effect of antifungal hot-water treatments on papaya postharvest quality and activity of pectin methylesterase and polygalacturonase. Journal of Food Technology, 50(1), 101-107.
Chen, N.M. and Paull, R.E. 1986. Development and prevention of chilling injury in papaya fruit. J. Amer. Soc. Hort. Sci. 111, 639-643.

Eaks, I.L. 1985. Effect of calcium on ripening respiratory rate, ethylene production, and quality of avocado fruit. J. Amer. Soc. Hort. Sci. 110(2), 145-148.

Fante C.A., Vilas, A.C., Aguiar, V.P., Freitas, C.R. and Carlos, L.O. 2014. Modified atmosphere efficiency in the quality maintenance of Eva apples Food Sci. Technol, Campinas, 34(2), 309-314.

Flores, F.B., Martínez-Madrid, M.C., Amor, M.B., Pech, J.C., Latché, A. and Romojaro, F. 2004. Modified Latmosphere packaging confers additional chilling tolerance on ethyleneinhibited cantaloupe Charentais melon fruit. European Food Research and Technology, 219(6), 431-435.

Fonseca, S.C., Oliveira, F.A. and Brecht, J.K. 2002. Modelling respiration rate of fresh fruits and vegetables for modified atmosphere packages: a review. Journal of Food Engineering, 52(2), 99-119.

Gallerani, G., Pratella, G., Bertolini, P. and Marchi, A. 1990. Lake of relationship between total calcium of apple fruit and a calcium deficiency related disorder (bitter pit): A four year report. Acta Hortic., 274, 141-148.

Gonzalez G.A., Buta, J.G. and Wang, C.Y. 2001. Methyl jasmonate reduces chilling injury symptoms and enhances colour development of 'Kent' mangoes Sci Food Agric., 81, 12441249.

González G.A., Buta, J.G. and Wang, C.Y. 2003. Methyl jasmonate and modified atmosphere packaging (MAP) reduce decay and maintain postharvest quality of papaya 'Sunrise' Posth Biol Technol.; 28, 361-370.

Grove, T., De-Beer, M.S. and Steyn, W.P. 2000. Further evaluation of heat shock treatments to develop tolerance to quarantine cold treatments. S. Afr. Avocado Growers AssoYearbook, 23, 103-108.

Guan, J.F. and Dou, S. 2010. The effect of MAP on quality and browning of cold-stored plum fruits. Journal of Food, Agriculture and Environment, 8(2), 113-116.

Hernandez-Munoz, E., Almenar, M.J. and Gavara, O.R. 2006. Effect of calcium dips and chitosan coating on postharvest life of strawberries (Fragariaxananassa). Postharvest Biol. Technol., 39, 247-253. 
Ismail, A., Lamia, H., Mohsen, H. and Bassem, J. 2011. Chemical composition of Juniperus oxycedrus L. subsp Macrocarpa essential oil and study of their herbicidal effects on germination and seedling growth of weeds. Asian J. Applied Sci., 4, 771-779.

Kader, A.A. 2002. Postharvest Technology of Horticultural Crops. Third Ed., Univ. Cal., Agric. Natl. Res., Publ. 3311.

Koukounaras, T.A., Lagopodi, L.K. and Sfakiotakis, C.E. 2008. The Effect of Post-storage Hot Water Dipping on Control of Botrytis cinerea Rot and on Preservation of Quality of'Hayward' Kiwifruit. Fresh Produce 2(1), 2631.

Laufmann, J.E. and Sams, C.E. 1989. The effect of postharvest calcium treatment on polyphenol oxidase and peroxidase activity in golden delicious apples. HortScience., 24, 753-754.

Lazan, H., Ali, Z.M. and Sim, W.C. 1990. Retardation of ripening and development of water stress in papaya seal packaged with polyethylene film. Acta Hortcult., 269, 345-351.

Lederman, E., Zauberman, G., Weksler, A., Rot, I. and Fuchs, Y. 1997. Ethylene forming capacity cold storage and chilling injury development in "Keitt" mango of fruit. Postharvest Biology and Technology, 10, 107-112.

Lelièvre, J.M., Tichit, L., Fillion, L., Larrigaudière, C., Vendrell, M. and Pech, J.C. 1995. Cold-induced accumulation of 1aminocyclopropane-1-carboxylate oxidase protein in Granny Smith apples. Postharv. Biol. Technol. 5, 11-17.

Lester, G.E. and Grusak, M.A. 2004. Field application of chelated calcium: Postharvest effects on muskmelon and honeydew fruit quality. HortTechnology 14, 29 - 38.

Lester, G., Dunlapa, J. and Lingle, S. 1988. Effect of postharvest heating on electrolyte leakage and fresh weight loss from stored muskmelon fruit . Hort. Science. 23, 407

Lurie S. 1998. Postharvest heat treatments of horticultural crops. Hortic Rev 22, 91- 121.

Lurie, S., Pesis, E., Gadiyeva, O., Feygenberg, O., Ben-Arie, R., Kaplunov, T., Zutahy, Y. and Lichter, A. 2006. Modified ethanol atmosphere to control decay of table grapes during storage. Postharvest Biology and Technology, 42(3), 222-227.

Maharaj, R. and Sankat, C.K. 1990. Storability of papayas under refrigerated and controlled atmosphere. Acta Hort. 269, 375-386.
Mahmud, T.M., Al-Eryani-Raqeeb, A., Syed Omar, S.R. and Mohamed Zaki, A.R. 2008. Effects of Different Concentrations and Applications of Calcium on Storage Life and Physicochemical Characteristics of Papaya (Carica Papaya L.) American Journal of Agricultural and Biological Sciences 3(3), 526-533.

Mathooko, F.M. 1996. Regulation of ethylene biosynthesis in higher plants by carbon dioxide. Postharvest Biology and Technology, 7(1-2), 1-26.

McCollum, T.G. and McDonald, R.E. 1993. Tolerance of cucumber fruit to immersion in heated water and subsequent effects on chilling tolerance. Acta Hort., 343, 233-237.

Morris, E.R. 1980. Physical probes of polysaccharide conformations and interactions. Food Chem., 6, 15-39.

Nasr, S.I. 2004. Studies on reducing mango fruits sensitivity to chilling injury. Ph.D. Thesis, Dept. Hort., Fac. Agric., Ain Shams Univ., Cairo, Egypt.

Paull, R. 1990. Chilling Injury of Crops of Tropical and Subtropical Origin. In: Chilling Injury of Horticultural Crops.

Paull, R.E., Nishijima, W., Reyes, M. and Cavaletto, C. 1997. Postharvest handling and losses during marketing of papaya (Carica papaya L.). Postharvest Biol. Technol., 11, 165-179.

Poovaiah, B.W., Glenn, G.M. and Reddy, A.S.N. 1998. Calcium and fruit softening: physiology and biochemistry. Horticultural Reviews, 10, 107-152.

Rai, D.R., Chadha, S., Kaur, M.P., Jaiswal, P. and Patil, R.T. 2011. Biochemical, microbiological and physiological changes in Jamun (Syzyiumcumini L.) kept for long term storage under modified atmosphere packaging. Journal of Food Science and Technology, 28(3), 357-365.

Rocha, A.M., Barreira, M. and Morais, A.M. 2004. Modified atmosphere package for apple 'Bravo de Esmolfe'. Food Control, 15(1), 6164.

Rohani, M.Y., Zaipun, M.Z. and Norhayati, M. 1997. Effect of modified atmosphere on the storage life and quality of Eksotika papaya. J. Trop. Agri.c Food Sc i., 25, 103-113.

Sabehat, A., Lurie, S. and Weiss, D. 1998. Expression of small heat-shock proteins at low temperatures: A possible role in protecting against chilling injuries. Plant Physiol., 117, 651-658. 
Sams, C.E., Conway, S.W., Abbott, J.A., Lewis, R.J. and Benshalom, N. 1993. Firmness and decay of apples following postharvest pressure infiltration of calcium and heat treatment. $\mathbf{J}$. Am. Soet Horde. Set., 118, 623-627.

Van-Eeden, S.J. 1992. Calcium in filtration as a possible post-harvest treatment to increase storage potential of mango fruit. Yearbook. South. African Mango Growers. Association. 12, 26-27.

Wang, C.Y. 1994. Chilling injury of tropical horticultural commodities. Hort Science, 29, 986-988.
Woolf, A.B., Watkins, C.B., Bowen, J.G., Maindonald, J.H., Lay-Yaa, M. and Ferguson, C.B. 1995. Reducing external chilling injury in stored "Hass avocados with dry heat treatments. J. Amer. Soc. Hort. Sci., 120, 10501056.

Yang, S.F. and Hoffman, N.E. 1984. Ethylene biosynthesis and its regulation in higher plants. Ann. Rev. Plant Physiol., 35, 155-189.

Steel R.G., Torrie, J.H. and Dickey, D.A. 1997. Principles and procedures of statistics; a biometrical approach. 3. Boston: McGraw-Hill. 